TRANSACTIONS OF THE

AMERICAN MATHEMATICAL SOCIETY

Volume 354, Number 4, Pages 1473-1485

S 0002-9947(01)02896-3

Article electronically published on November 20, 2001

\title{
WANDERING ORBIT PORTRAITS
}

\author{
JAN KIWI
}

\begin{abstract}
We study a counting problem in holomorphic dynamics related to external rays of complex polynomials. We give upper bounds on the number of external rays that land at a point $z$ in the Julia set of a polynomial, provided that $z$ has an infinite forward orbit.
\end{abstract}

\section{INTRODUCTION}

We study external rays which land at a common point in the Julia set $J(f)$ of a polynomial $f$. Our main result gives an upper bound on the number of external rays that land at a point $z \in J(f)$ with infinite forward orbit.

For quadratic polynomials with connected Julia sets, by Thurston's work [T] on quadratic laminations, at most 4 rays land at a point $z$ with infinite forward orbit. Moreover, all but finitely many forward images of a point are landing points of at most 2 rays (see Theorem II.5.2 in [Th]). We generalize Thurston's result as follows:

Theorem 1.1. Consider a monic polynomial $f$ of degree $d \geq 2$ with connected Julia set $J(f)$. Let $z \in J(f)$ be a point with infinite forward orbit $\mathcal{O}(z)=\left\{f^{\circ n}(z)\right\}_{n \geq 0}$. Then the number of external rays landing at $z$ is at most $2^{d}$. Moreover, if $\mathcal{O}(z)$ is disjoint from the critical points of $f$, then the number of external rays landing at $z$ is at most $d$.

For quadratic polynomials, both bounds are sharp and due to Thurston. For higher degree polynomials, we expect $2^{d}$ to be optimal in the statement of the theorem. However, we do not know whether or not there is an infinite forward orbit of a cubic polynomial with exactly 3 external rays landing at each orbit element.

The results contained in this article are part of the author's thesis [Ki1]. Recently Blokh and Levin $[\mathrm{L}, \mathrm{BL}$ introduced a new set of ideas leading to related results. They consider the more general problem of counting the number of external rays that land at a collection of points with distinct infinite forward orbit. For a single point, their bounds coincide with those of Theorem 1.1 Blokh, Levin and Thurston work in the more abstract setting of "invariant laminations" (see [D, BL, Ke, Th]). From Theorem 1.1, it is not difficult to obtain an abstract result which also applies to "invariant laminations" (see Theorem A.1 in Ki2 ).

To give the corresponding discussion for points with finite forward orbit we also assume that $J(f)$ is connected. Points with finite forward orbit are either

Received by the editors April 11, 2000 and, in revised form, March 29, 2001.

2000 Mathematics Subject Classification. Primary 37F10, 37F20.

Supported by "Proyecto Fondecyt \#1990436", "Fundación Andes, Chile" and "Cátedra Presidencial en Geometría". 
pre-periodic or periodic. The relevant discussion is the one for periodic points. There are three types of periodic points in the Julia set of a polynomial: repelling, parabolic, and Cremer points. At a repelling or parabolic point $z$ there is at least one and at most a finite number of external rays landing (e.g. see Section 18 in [M1]). There is no uniform bound for the number of external rays which land at $z$. That is, for any given number $N \geq 1$ and degree $d \geq 2$, there exists a degree $d$ polynomial $f$ with connected Julia set $J(f)$ and a parabolic or repelling periodic point $z \in J(f)$ such that $N$ external rays land at $z$. Nevertheless, in Section 3 we discuss some bounds related to external rays landing at repelling or parabolic periodic points. Very little is known about external rays landing at Cremer points. In fact, it is not known whether or not a ray can land at a Cremer point. If there exists a Cremer point with a ray landing at it, then the number of external rays landing at it is infinite.

It is worth to mention that according to Theorem 1.1 and well known results (e.g. see Section 18 in [M1]), there exists a point with infinitely many rays landing

at it if and only if there exists a Cremer point with infinitely many rays landing at it.

The main ingredients in the proof of Theorem 1.1 are the ideas and techniques introduced by Goldberg and Milnor [G, GM, M2] in order to study external rays which land at a common point. Our proof may be regarded as a generalization of Thurston's argument using the language of Goldberg and Milnor.

This paper is organized as follows:

All of the polynomials considered in this article are monic of degree at least 2 . We deal with polynomials which have connected Julia sets except in Appendix A where polynomials with disconnected Julia sets are considered.

We recall some definitions and results from complex polynomial dynamics in Section 2. Also, following Goldberg and Milnor GM M2, we discuss the basic objects and results in order to study external rays which land at a common point; that is, we introduce types and sectors, as well as their fundamental properties. In Section 3 we apply these properties to obtain bounds on the number of cycles of external rays which land at a periodic orbit. These bounds are a straightforward generalization of a result by Milnor [M2]. The arguments used in Section 3 illustrate the ideas behind the proof of our main result. Section 4 will be devoted to the proof of the main theorem, Theorem 1.1 Similar results for polynomials with disconnected Julia sets are discussed in Appendix A.

\section{Preliminaries: External Rays, SeCtors and Angular size}

We begin by recalling some definitions and results from complex polynomial dynamics. For background material on holomorphic dynamics, the reader can consult [CG, M2] and the references therein. In Paragraphs 2.2 2.3 and 2.5] following Goldberg and Milnor, we introduce some notions (orbit portraits, sectors, angular size, etc.) which are helpful in counting external rays that land at a common point. In Paragraph 2.4 we give an example which illustrates the objects and results presented in Paragraphs 2.2, 2.3 and 2.5.

2.1. External Rays. In what follows, we assume that the polynomials under consideration are monic of degree $d \geq 2$.

The basin of infinity $\Omega(f)$ of a polynomial $f: \mathbb{C} \rightarrow \mathbb{C}$ with connected Julia set $J(f)$ is conformally isomorphic to the complement of the closed unit disk $\overline{\mathbb{D}}$. Since 
$f$ is monic, there is a preferred choice of conformal isomorphism $\phi: \Omega(f) \rightarrow \mathbb{C} \backslash \overline{\mathbb{D}}$ which is asymptotic to the identity at $\infty$ and conjugates $f$ with $z \mapsto z^{d}$. That is, $\phi(f(z))=\phi(z)^{d}$. The preimage of a half line $\left\{r e^{2 \pi i t}: r>1\right\}$ under the Böttcher map $\phi$ is a smooth arc called the external ray $R^{t}$ with argument $t \in \mathbb{R} / \mathbb{Z}$. This smooth arc approaches the Julia set at one end and infinity at the other. If it has a well defined limit $z$ as it approaches the Julia set we say that $R^{t}$ lands at $z$. Note that, under $f$, the external ray $R^{t}$ maps diffeomorphically onto the external ray $R^{d t}$. (See Section 18 in M1.)

\subsection{Types and Sectors.}

Definition 2.1. Consider a point $z$ in a connected Julia set $J(f)$, which is the landing point of at least one ray. The type $A(z)$ of $z$ is the subset of $\mathbb{R} / \mathbb{Z}$ formed by the arguments of the external rays landing at $z$.

Let $\mathcal{O}=\{z, f(z), \ldots\}$ be the forward orbit of $z$. We say that

$$
A(\mathcal{O})=\{A(w): w \in \mathcal{O}\}
$$

is the orbit portrait of $\mathcal{O}$. In particular, when $\mathcal{O}$ is a periodic cycle, $A(\mathcal{O})$ is called a periodic orbit portrait, and when $\mathcal{O}$ is infinite, $A(\mathcal{O})$ will be called a wandering orbit portrait.

Here and throughout, $d \cdot A$ will denote the image of a subset $A$ of $\mathbb{R} / \mathbb{Z}$ under the "multiplication by $d$ " map $m_{d}$. Notice that $A(f(z))=d \cdot A(z)$. Moreover, if $d_{z}$ is the local degree of $f$ at $z$, then multiplication by $d$ maps $A(z)$ onto $d \cdot A(f(z))$ in a $d_{z}$-to-one fashion.

We want to count the number of external rays that participate in some types. Following Goldberg and Milnor [GM, M2], several counting problems may be tackled through a detailed study of the partitions of $\mathbb{C}$ and $\mathbb{R} / \mathbb{Z}$ which arise from a given type. In order to obtain useful information, we work under the assumption that there are finitely many elements participating in a given type $A(z)$. The fact that almost all types are finite will follow from Theorem 1.1 and well known landing theorems (e.g., see Theorems 18.10 and 18.11, as well as Lemma 18.12 in [M1]). More precisely, the only types that have a chance of being infinite are types of Cremer points and pre-Cremer points. However, it is not known whether or not there exists a Cremer point with a ray landing at it.

Definition 2.2. Consider a point $z \in J(f)$ with type $A(z)$ that has finite cardinality. Let $\Gamma$ be the graph formed by the union of the external rays landing at $z$ and the singleton $\{z\}$. A connected component $S$ of $\mathbb{C} \backslash \Gamma$ is called a sector based at $z$.

The global image under $f$ of a sector based at $z$ is not necessarily a sector based at $f(z)$. However, locally around $z, f$ maps sectors onto sectors.

Definition 2.3. For a type $A(z)$ with finite cardinality, we define a map $\tau$ which assigns to each sector based at $z$, a sector based at $f(z)$ as follows. Given a sector $S$ based at $z$, let $\tau S$ be the unique sector based at $f(z)$ such that $f(S \cap V) \subset \tau S$, for some neighborhood $V$ of $z$. We call $\tau$ the local sector map at $z$.

2.3. Angular Size and Critical Points. While types live in $\mathbb{R} / \mathbb{Z}$, external rays are contained in the complex plane $\mathbb{C}$. It is convenient to have both objects in the same topological space: 
Definition 2.4. The circled plane (c) is the closed topological disk obtained by compactifying $\mathbb{C}$ with a circle of points $\lim _{r \rightarrow \infty} r e^{2 \pi i t}$ at infinity. The boundary $\partial(C)$ is canonically identified with $\mathbb{R} / \mathbb{Z}$.

When a sector $S$ is regarded as a subset of the circled plane (C), the portion of $\partial S$ contained in the circle at infinity $\mathbb{R} / \mathbb{Z} \cong \partial \subseteq$ is an interval $I$. The angular size $\alpha(S)$ of $S$ is defined as the length of the interval $I$. Here, the total length of $\mathbb{R} / \mathbb{Z}$ is 1 .

The basic relations among the number of critical points and values contained in a sector, angular size, and sector dynamics are included in the next lemma, which, in a slightly more general setting, is a compilation of results due to Goldberg and Milnor.

Lemma 2.5. Let $S$ be a sector, and let $\omega(S)$ be the number of critical points (counting multiplicities) contained in $S$. Then:

(a) $\alpha(\tau S)+\omega(S)=d \alpha(S)$. Thus $\omega(S)$ is the largest integer strictly less than $d \alpha(S)$.

(b) If $\omega(S)=0$ (or equivalently $\alpha(S) \leq 1 / d$ ), then $f$ maps $S$ homeomorphically onto $\tau S$. Moreover, $\alpha(\tau S)=d \alpha(S)$.

(c) If $\omega(S)>0$, then $\tau S$ contains at least one critical value.

(d) If $\alpha(\tau S) \leq \alpha(S)$, then $\tau S$ contains at least one critical value.

For the proof, we refer the reader to the original papers $[\mathrm{G}, \mathrm{GM}]$. More precisely, for (a) see Lemma 3 in [G] or 2.7 in [GM], for (b) see Lemma 2.5 in [GM], for (c) see Remark 2.6 in [GM] and, finally, (d) follows immediately from (b) and (c). Another explanation for these results may be found in sections 3 and 4 of the author's thesis Ki1.

2.4. Example. Consider the cubic polynomial

$$
f(z)=z^{3}+(-.05789039625+.01400272469 i) z-.6082787557-.6715881263 i,
$$

with critical points

$$
\omega_{ \pm}= \pm(.1399108252-.01668053522 i)
$$

both being periodic of period 4 and in distinct orbits.

The immediate basins of attraction of the critical points have a common boundary point $z_{0}$, which is periodic of period 4 with type

$$
A\left(z_{0}\right)=\{1 / 5,43 / 80,69 / 80\} .
$$

The corresponding orbit portrait $A\left(\mathcal{O}\left(z_{0}\right)\right)$ is

$$
\begin{aligned}
& \{\{1 / 5,43 / 80,69 / 80\},\{3 / 5,49 / 80,47 / 80\}, \\
& \{4 / 5,67 / 80,61 / 80\},\{2 / 5,41 / 80,23 / 80\}\} .
\end{aligned}
$$

In Figure 1, we illustrate a sector $S$ based at $z_{0}$. This sector has angular size $\alpha(S)=0.3375>1 / 3$ and contains the critical point $\omega_{+}$, thus the critical weight $\omega(S)=1$. The image $\tau S$ under the local sector map has angular size $\alpha(S)=3 \times 0.3375-1=0.0125$. Moreover, $\tau S$ contains the critical value $f\left(\omega_{+}\right)$. The sectors $\tau^{\circ 2} S$ and $\tau^{\circ 3} S$ are also illustrated in Figure 1 Note that $S=\tau^{\circ 4} S$. 

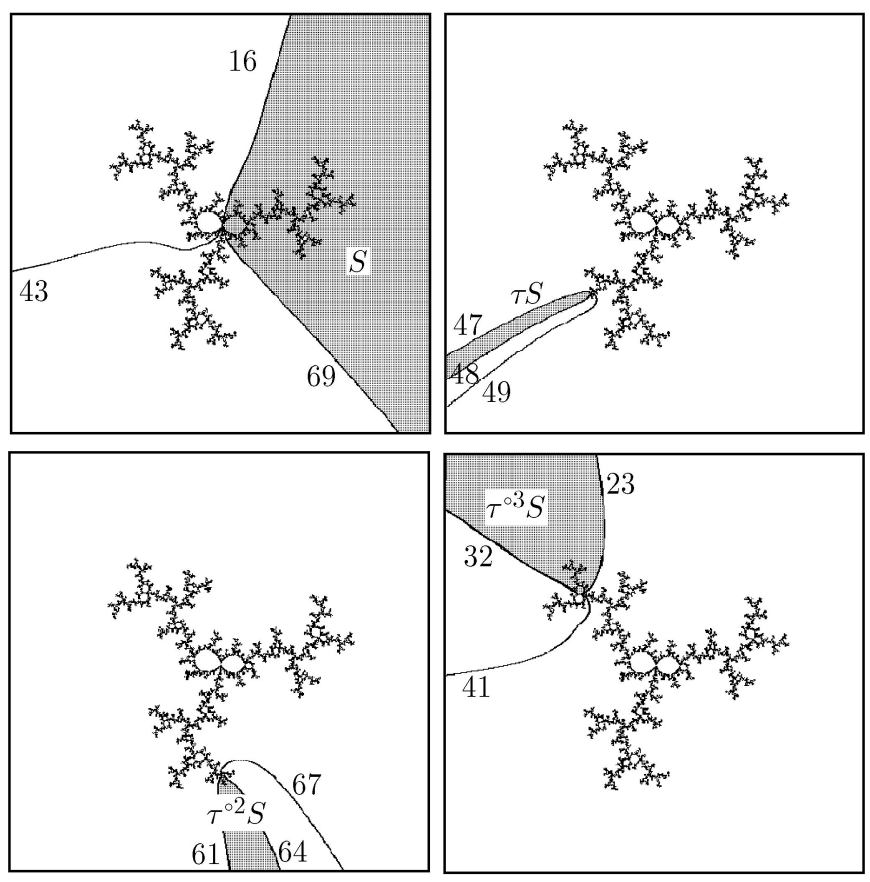

Figure 1. Illustration of the example discussed in Paragraph 2.4 The arguments of external rays are labeled taking $1 / 80$ of a turn as the unit of measurement.

2.5. Types and Sectors Based at Distinct Points. The disjointness of external rays imposes certain restrictions on how types of distinct points are distributed in $\mathbb{R} / \mathbb{Z}$ and sectors based at distinct points are distributed in $\mathbb{C}$.

The reader might find Figure 2 useful to visualize the distribution of the four types and the twelve sectors involved in the example discussed in Paragraph 2.4.

To describe the above-mentioned restrictions we need the following definition:

Definition 2.6. We say that two disjoint finite subsets $B_{1}$ and $B_{2}$ of $\mathbb{R} / \mathbb{Z}$ are unlinked if $B_{1}$ is contained in a connected component of $\mathbb{R} / \mathbb{Z} \backslash B_{2}$.

Equivalently, $B_{1}$ and $B_{2}$ are unlinked if there exists disjoint intervals $I_{1}$ and $I_{2}$ in $\mathbb{R} / \mathbb{Z}$ such that $I_{1}$ contains $B_{1}$ and $I_{2}$ contains $B_{2}$. Also equivalently, $B_{1}$ and $B_{2}$ are unlinked if, after identifying $\mathbb{R} / \mathbb{Z}$ with the boundary $\partial \mathbb{D}$ of the unit disk $\mathbb{D}$, the convex hulls of $B_{1}$ and $B_{2}$, with respect to the Euclidean or Poincaré metric in $\mathbb{D}$, are disjoint.

Let us consider two distinct points $z$ and $\hat{z}$ with types $A(z)$ and $A(\hat{z})$ with finite cardinality.

We claim that the types $A(z)$ and $A(\hat{z})$ are unlinked. In fact, observe that $z$ belongs to a sector $\hat{S}$ based at $\hat{z}$. Hence, all the rays landing at $z$ are contained in that sector $\hat{S}$. Therefore, the interior of the boundary of $\hat{S}$ in the circle at $\infty$ contains $A(z)$. That is, a connected component of $\mathbb{R} / \mathbb{Z} \backslash A(\hat{z})$ contains $A(z)$.

Now consider a sector $S$ based at $z$ and a sector $\tilde{S}$ based at $\hat{z}$. It follows that $S$ and $\tilde{S}$ are either nested, or disjoint, or one contains the complement of the other. 


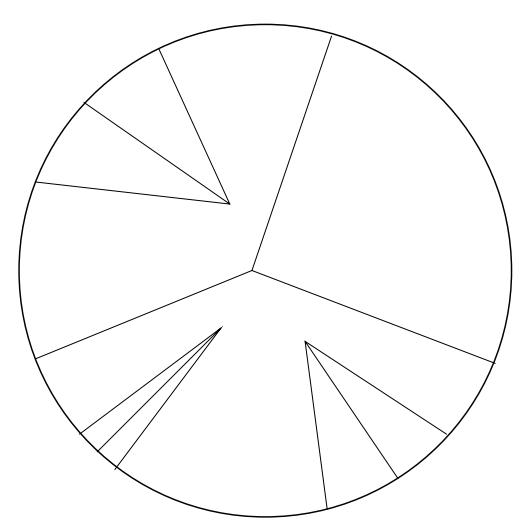

Figure 2. Schematic diagram.

\section{Periodic orbit portraits}

In this section, we study types of periodic points assuming that there are periodic rays landing at these points. Note that the only periodic points in the Julia set at which no periodic ray lands are Cremer points. We give an upper bound on the number of cycles of rays which land at a periodic orbit. Here the results are an immediate generalization of those obtained by Milnor for quadratic polynomials (see [M2]). In the next section, we apply similar ideas in order to give an upper bound on the number of external rays landing at a point with infinite forward orbit.

To a periodic orbit portrait, we assign a rotation number as follows. Let $\mathcal{O}=\left\{z_{0}, f\left(z_{0}\right), \ldots, f^{\circ(p-1)}\left(z_{0}\right)\right\} \subset J(f)$ be a periodic cycle of period $p$ with portrait $A(\mathcal{O})$ formed by periodic arguments. That is, $\mathcal{O}$ is a parabolic or repelling cycle and each point in $\mathcal{O}$ the landing point of periodic rays of the same period. The return map to $A\left(z_{0}\right)$ given by $t \mapsto d^{p} t$ is cyclic order preserving. Thus the return map has a well defined rotation number $\operatorname{rot} A(\mathcal{O}) \in \mathbb{Q} / \mathbb{Z}$ which does not depend on the choice of $z_{0} \in \mathcal{O}$. The rotation number of $A(\mathcal{O})$ is also called the combinatorial rotation number of $\mathcal{O}$.

For a quadratic polynomial, Milnor [M2 shows that the number of cycles of rays landing at a periodic orbit $\mathcal{O}$ is at most 2. Moreover, if the number of cycles is 2, then $\mathcal{O}$ has zero combinatorial rotation number. Our next theorem generalizes this result.

Theorem 3.1. Let $\mathcal{O}$ be a periodic orbit of a degree $d$ polynomial $f$ with connected Julia set. The number of cycles of external rays landing at $\mathcal{O}$ is at most $d$. Moreover, if the number of cycles is $d$, then $\mathcal{O}$ has zero combinatorial rotation number.

These bounds are obtained by showing that the number of cycles gives rise to a lower bound on the number of critical values of the polynomial under consideration. Hence we prefer to state the result as follows.

Theorem 3.2. If a polynomial $f$ with connected Julia set has exactly $k$ distinct critical values, then the number of cycles of external rays landing at a periodic orbit $\mathcal{O}$ is at most $k+1$. Moreover, if the number of cycles is $k+1$, then $\mathcal{O}$ has zero combinatorial rotation number.

Remark 3.3. The bounds on the number of cycles are sharp. In fact, every parabolic periodic orbit $\mathcal{O}$, with $d-1$ immediate basins and multiplier other than 1 , 
has exactly $d-1$ cycles of rays which land at $\mathcal{O}$. In this case, $\mathcal{O}$ has nonzero combinatorial number.

Figure 1, which illustrates the dynamical plane of the polynomial considered in Paragraph [2.4, shows a cubic periodic orbit with three cycles of rays landing at it and zero combinatorial rotation number.

To prove the theorem we need the next result (cf. [M2]) which shows that the smallest sector in a cycle of sectors based at $\mathcal{O}$ contains a critical value:

Lemma 3.4. Let $\mathcal{O}$ be a periodic orbit with at least one periodic ray landing at each orbit point. Let $S$ be a sector based at $\mathcal{O}$ and $\tau$ the local sector map at $\mathcal{O}$. If $\alpha(S)=\min \left\{\alpha\left(\tau^{\circ n} S\right): n \in \mathbb{N}\right\}$, then $S$ contains at least one critical value.

Remark 3.5. Notice that, if $\mathcal{O}$ is a periodic orbit as in the lemma, then the local sector map $\tau$ at $\mathcal{O}$ is a well defined permutation of the sectors based at $\mathcal{O}$. Remark that the number of cycles of sectors under $\tau$ coincides with the number of cycles of external rays landing at $\mathcal{O}$.

Proof of Lemma 3.4. Consider a sector $S$ with minimal angular size in its cycle. If $S$ does not contain critical values, then Lemma 2.5 (c) shows that $w\left(\tau^{-1} S\right)=0$. By Lemma 2.5 (b), we have $\alpha\left(\tau^{-1} S\right)=\alpha(S) / d$, which contradicts the minimality of the angular size.

Proof of Theorem 3.2. Suppose that $A(\mathcal{O})$ has $n$ cycles. It follows that there are exactly $n$ cycles $C_{1}, \ldots, C_{n}$ of sectors based at $\mathcal{O}$. Let $S_{i}$ be a sector with minimal angular length in its cycle $C_{i}$. Changing subscripts, if necessary, we may assume that

$$
\alpha\left(S_{1}\right) \leq \cdots \leq \alpha\left(S_{n}\right) .
$$

Suppose that $\operatorname{rot} A(\mathcal{O}) \neq 0$ and that $n>k$. We show that this assumption yields a contradiction. According to Lemma $3.4 S_{i}$ contains a critical value. Thus to obtain a contradiction, it suffices to show that $S_{1}, \ldots, S_{k+1}$ are pairwise disjoint.

In effect, suppose that $1 \leq i<j \leq k+1$, and $S_{i} \cap S_{j} \neq \emptyset$. Then either $S_{i}$ contains the basepoint $z$ of $S_{j}$ or $S_{j}$ contains the basepoint $w$ of $S_{i}$. Without loss of generality, assume the first case. In this case, $S_{i}$ contains all of the sectors based at $z$ except for one. Since $C_{i}$ has at least 2 sectors based at $z$ (nonzero rotation number), it follows that $S_{i}$ properly contains a sector in its cycle $C_{i}$. This contradicts the minimality of the angular size of $S_{i}$ in its cycle of sectors.

Next suppose that $\operatorname{rot} A(\mathcal{O})=0$ and that $n>k+1$. Again, we obtain a contradiction by showing that $S_{1}, \ldots, S_{k+1}$ are pairwise disjoint.

Suppose that $1 \leq i<j \leq k+1$ and $S_{i} \cap S_{j} \neq \emptyset$. Then either $S_{i}$ contains the basepoint $z$ of $S_{j}$ or $S_{j}$ contains the basepoint $w$ of $S_{i}$. In the first case, $S_{i}$ contains all of the sectors based at $z$ except for one, which must be in the cycle of $S_{i}$. Therefore $S_{i}$ properly contains at least $k+1$ sectors that are in different cycles, thus contradicting $i \leq k+1$. The second case is identical.

\section{WANDERING ORBIT PORTRAITS}

This section is devoted to the proof of Theorem 1.1 that is, we give an upper bound on the number of external rays which land at a point $z_{0}$ with infinite forward orbit. We accomplish this by showing that the number of critical values of $f$ gives rise to a bound on the number of external rays landing at $z_{0}$. 
In Paragraph 4.1, we distinguish between two types of critical values. That is, we introduce the notions of "narrow" and "wide" critical values. These notions allow us to state our main lemma which shows that the number of narrow critical values gives rise to an upper bound on number of rays landing at certain points. Also in Paragraph 4.1 we show that Theorem 1.1 is a consequence of this main lemma (Lemma 4.3). Paragraph 4.2 contains the proof of the main lemma (Lemma 4.3).

4.1. Narrow and Wide Critical Values. A priori we do not know if only finitely many rays land at a point $z_{0}$. Hence we are forced to work with finite subsets of the type of $z_{0}$. Recall that the type $A(z) \subset \mathbb{R} / \mathbb{Z}$ of a point $z \in J(f)$ is the set that consists of the arguments of the external rays which land at $z$.

More precisely, consider a point $z \in J(f)$ and a finite subset $A \subset A(z)$ of the type of $z$. The graph formed by the union of the external rays, with arguments in $A$, with $\{z\}$ cuts the complex plane into $|A|$ sectors based at $z$ (here $|A|$ denotes the cardinality of $A$ ). When necessary, a sector as above will be called with respect to $A$. Although we may be working with a proper subset of the rays which land at $z$, the definition of angular size easily generalizes (Definition 2.4).

Intuitively, a critical value $v$ is "narrow" if $v$ is contained in arbitrarily small sectors. Formally:

Definition 4.1. Fix a point $z_{0}$, with infinite forward orbit $\mathcal{O}\left(z_{0}\right)=\left\{f^{\circ n}\left(z_{0}\right)\right\}_{n \geq 0}$ that is disjoint from the critical points of $f$. Consider the collection $\mathcal{S}$ which consists of all the sectors based at $\mathcal{O}\left(z_{0}\right)$. Given a critical value $v$, let width $(v)$ denote the infimum of the angular size of the sectors $S \in \mathcal{S}$ which contain $v$.

We say a critical value $v$ is narrow if $\operatorname{width}(v)=0$. Otherwise, we will say $v$ is a wide critical value.

Our definition of narrow and wide critical values depends on the initial choice of the point $z_{0}$. That is, a critical value $v$ may be narrow with respect to an initial choice $z_{0}$ but wide with respect to an initial choice $z_{0}^{\prime}$.

Remark 4.2. A critical value which is the landing point of two or more rays is automatically a wide critical value.

We may now state the inequality which relates the cardinality of $A\left(z_{0}\right)$ with the number $\nu$ of narrow critical values in the following result.

Lemma 4.3 (Main). Let $f$ be a polynomial with connected Julia set $J(f)$. Consider a point $z_{0} \in J(f)$, with infinite forward orbit $\left\{z_{n}=f^{\circ n}\left(z_{0}\right)\right\}_{n \geq 0}$ that is disjoint from the critical points of $f$, such that $\left|A\left(z_{0}\right)\right| \geq 3$. If $\nu$ is the number of narrow critical values of $f$, then

$$
\nu \geq\left|A\left(z_{0}\right)\right|-1
$$

We prove the above result in Paragraph 4.2. Our next theorem is a slightly improved version of Theorem 1.1 .

Theorem 4.4. Consider a monic polynomial $f$ of degree $d$, with connected Julia set $J(f)$ and $\delta$ distinct critical values. Let $z \in J(f)$ be a point with infinite forward orbit $\mathcal{O}(z)=\left\{f^{\circ n}(z)\right\}_{n \geq 0}$. Then the number of external rays landing at $z$ is at most $2^{d}$. Moreover, if $\mathcal{O}(z)$ is disjoint from the critical points of $f$, then the number of external rays landing at $z$ is at most $\delta+1$.

The proof follows from Lemma 4.3 
Proof of Theorem 4.4. Given a point $z_{0} \in J(f)$ with infinite forward orbit $\mathcal{O}\left(z_{0}\right)=$ $\left\{z_{n}=f^{\circ n}\left(z_{0}\right)\right\}$. Let $z_{j_{1}}, \ldots, z_{j_{a}}$, with $j_{1}<\cdots<j_{a}$, be the critical points of $f$ which lie in $\mathcal{O}\left(z_{0}\right)$. We let $d_{1}, \ldots, d_{a}$ denote the corresponding local degrees. By Lemma 4.3, $\left|A\left(z_{j_{a}+1}\right)\right| \leq \nu+1$ where $\nu$ is the number of narrow critical values of $f$. Hence

$$
\left|A\left(z_{0}\right)\right| \leq d_{1} \cdots d_{a} \cdot(\nu+1) .
$$

According to Remark 4.2 $f\left(z_{j_{1}}\right), \ldots, f\left(z_{j_{a}}\right)$ are wide critical values. We conclude that the sum $d_{1}+\cdots+d_{a} \leq d-1-\nu+a$. Since $d_{k} \geq 2$, it follows that $d_{1} \cdots d_{a} \leq$ $2^{d-1-\nu}$. Therefore

$$
d_{1} \cdots d_{a} \cdot(\nu+1) \leq 2^{d-1-\nu} \cdot(\nu+1) \leq 2^{d},
$$

which completes the proof.

Theorem 1.1 is an immediate consequence of Theorem 4.4. since a degree $d$ polynomial has at most $d-1$ distinct critical values.

4.2. Proof of the Main Lemma (Lemma 4.3). Throughout, distinct critical values which are not separated by rays landing at the orbit of $z_{0}$ are identified (i.e., counted as one critical value).

First we isolate each narrow critical value by choosing a collection of pairwise disjoint sectors, where each sector contains one and only one critical value. More precisely, a pairwise disjoint collection of sectors

$$
\{S(v) \in \mathcal{S}: v \text { is a narrow critical value }\}
$$

such that $v$ is the unique critical value in $S(v)$, and $\alpha(S(v))<1 / 2$, where $\mathcal{S}$ is the collection which consists of all sectors based at $\mathcal{O}\left(z_{0}\right)$.

Suppose there are $\mu+1 \geq 3$ rays landing at $z_{0}$. We let $A_{0}$ be the set formed by the arguments of these $\mu+1$ rays. The $\mu+1$ external rays with arguments in $A_{n}=d^{n} \cdot A_{0}$ land at $z_{n}$ and cut the complex plane into $\mu+1$ sectors. In what follows, we restrict our attention to these sectors. Let

$$
S_{n}^{1}, \ldots, S_{n}^{\mu+1}
$$

denote the sectors based at $z_{n}$, and let

$$
\alpha_{n}^{1}, \ldots, \alpha_{n}^{\mu+1}
$$

denote their corresponding angular size. The superscripts are chosen so that the sectors are ordered according to their "size", that is,

$$
\alpha_{n}^{1} \leq \cdots \leq \alpha_{n}^{\mu+1} .
$$

Our map $f$ is a local homeomorphism at $z_{n}$. Thus it induces a bijection $\tau$ from the $\mu+1$ sectors based at $z_{n}$ onto the $\mu+1$ sectors based at $z_{n+1}$. Lemma 2.5 which establishes the basic relations among the critical points and values, the local sector map $\tau$, and the angular size, holds in this context as well.

After identifying $\mathbb{R} / \mathbb{Z}$ with $\partial \mathbb{D}$, we regard $A_{n}$ as a subset of $\partial \mathbb{D}$. Since the $A_{n}$ are pairwise unlinked (see Paragraph 2.5), the $(\mu+1)$-agons formed by their Euclidean convex hulls Convex $\left(A_{n}\right) \subset \overline{\mathbb{D}}$ are pairwise disjoint. To fit in $\overline{\mathbb{D}}$, the area of Convex $\left(A_{n}\right)$ must approach zero, as $n$ tends to infinity. We conclude that at most two sides of the $(\mu+1)$-agons $\operatorname{Convex}\left(A_{n}\right)$ can have length bounded away 


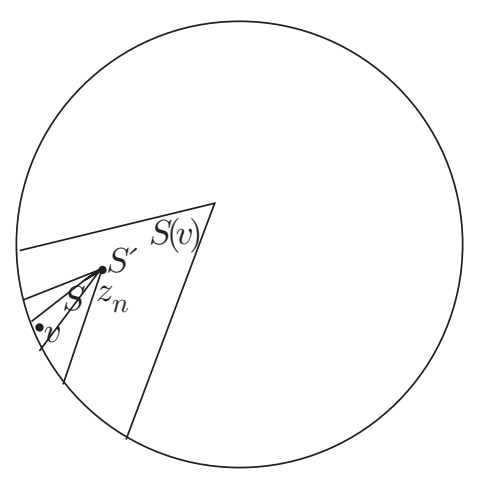

FIGURE 3.

from zero. Therefore the length of at least $\mu-1$ sides must tend to zero. Since the length of a side is comparable to the corresponding angular size, we have

$$
\lim _{n \rightarrow \infty} \alpha_{n}^{j}=0
$$

for $j=1, \ldots, \mu-1$.

We claim that there exist $n_{0}$ such that the smallest sector $S_{n_{0}}^{1}$ based at $z_{n_{0}}$ contains a narrow critical value $v_{0}$. Moreover, $n_{0}$ may be chosen so that $S_{n_{0}}^{1}$ has angular length $\alpha_{n_{0}}^{1}<1 / d$, with

$$
\alpha_{n_{0}}^{1} \leq \alpha(S(v)) \text {, for all narrow critical values } v,
$$

and

$$
\alpha_{n_{0}}^{1} \leq \alpha(S) \text {, for all sectors } S \text { that contain a wide critical value. }
$$

In fact, (1) implies the existence of an integer $M$ such that for all $n \geq M$ the three following inequalities hold:

(a) $\alpha_{n}^{1}<1 / d$,

(b) $\alpha_{n}^{1} \leq \alpha(S(v))$ for all narrow critical values $v$,

(c) $\alpha_{n}^{1} \leq \alpha(S)$ for all sectors $S$ that contain a wide critical value.

By Lemma 2.5 for some $n_{0} \geq M$ the sector $S_{n_{0}}^{1}$ must contain a critical value $v_{0}$, otherwise $\alpha_{n}^{1}$ would be an increasing function of $n$. From (c), it follows that the critical value $v_{0}$ must be narrow as desired.

We think of $\alpha_{n_{0}}^{1}$ as the threshold size. A sector of angular size greater than $\alpha_{n_{0}}^{1}$ is thought of as a big sector, and that of angular size smaller than $\alpha_{n_{0}}^{1}$ is thought of as a small sector.

If a small sector $S$ based at $z_{n}$ contains a critical value $v$, then (3) implies that $v$ is a narrow critical value, while (2) implies $S \subset S(v)$. Moreover, only one sector based at $z_{n}$ is not contained in $S(v)$. Thus only one sector $S^{\prime}$ based at $z_{n}$, other than $S$, may contain critical values. The sector $S^{\prime}$ is automatically big (see Figure 3).

The choice of a threshold size guarantees that when $n$ increases by 1 , the number of small sectors increases at most by 1 , that is,

$$
\alpha_{n-1}^{k-1} \geq \alpha_{n_{0}}^{1} \Longrightarrow \alpha_{n}^{k} \geq \alpha_{n_{0}}^{1} .
$$

In fact, if two big sectors $S$ and $S^{\prime}$ based at $z_{n-1}$ have small image sectors $\tau S$ and $\tau S^{\prime}$, respectively, then the small sectors $\tau S$ and $\tau S^{\prime}$ must contain critical values. This contradicts the previous discussion. 
For $1 \leq k \leq \mu-1$, let $n_{k}$ be the smallest integer greater than $n_{0}$ such that $\alpha_{n_{k}}^{k}<\alpha_{n_{0}}^{1}$. That is, $z_{n_{k}}$ is the first iterate after $z_{n_{0}}$ for which $k$ of the sectors based at $z_{n_{k}}$ are small. Note that the existence of such integers $n_{k}$ is guaranteed by (1). Moreover, according to (4) at most one sector at a time drops below the threshold size. Therefore, we have a strict inequality $n_{k+1}>n_{k}$.

There are at least two sectors $S_{n_{k}}^{\mu}$ and $S_{n_{k}}^{\mu+1}$ based at $z_{n_{k}}$ that are big. In fact, take $n=n_{k}$ in (4) and conclude that

$$
\alpha_{n_{k}}^{\mu+1} \geq \alpha_{n_{k}}^{\mu} \geq \alpha_{n_{k}}^{k} \geq \alpha_{n_{0}}^{1} .
$$

For each $1 \leq k \leq \mu-1$, there exists a big sector $S_{n_{k}-1}$ which maps onto a small sector $\tau S_{n_{k}-1}$. The sector $\tau S_{n_{k}-1}$ must contain a narrow critical value. Moreover, we claim that

$$
S_{n_{0}}^{1}, \tau S_{n_{1}-1}, \ldots, \tau S_{n_{\mu-1}-1}
$$

are pairwise disjoint. Otherwise, from this list, there exist two sectors $S$ and $S^{\prime}$ which are not disjoint. Either one of them is contained in the other, say $S \subset S^{\prime}$, or one of the sectors contains the complement of the other. The latter is impossible, since both $S$ and $S^{\prime}$ are small. The former implies that the small sector $S^{\prime}$ contains all but one of the sectors based at $z$, where $z$ is the base point of $S$. By (5), at least two sectors based at $z$ are big. Hence the small sector $S^{\prime}$ contains a big sector, which is impossible.

Thus each of the $\mu$ disjoint sectors

$$
S_{n_{0}}^{1}, \tau S_{n_{1}-1}, \ldots, \tau S_{n_{\mu-1}-1}
$$

contains a narrow critical value. Therefore

$$
\mu=\left|A_{0}\right|-1 \leq \nu
$$

The proof of Lemma 4.3 is now complete.

\section{Appendix A. Disconnected Julia sets}

When the Julia set $J(f)$ of a monic polynomial is disconnected, the basin of infinity is no longer conformally isomorphic to $\mathbb{C} \backslash \overline{\mathbb{D}}$. Nevertheless, external rays may be introduced. Following Appendix A of GM] (also compare with $[\mathrm{DH}]$ and $[\mathrm{At}]$ ), for an argument $t \in \mathbb{R} / \mathbb{Z}$, with the exception of those in a countable set $\Sigma \subset \mathbb{R} / \mathbb{Z}$, there exists a well defined external ray $R^{t}$. Non-exceptional external rays possess almost all of the properties which external rays of connected Julia sets have. In particular, they are disjoint smooth curves that approach infinity at one end and the Julia set at the other. Also, under $f$, a smooth external ray (i.e., non-exceptional) maps diffeomorphically onto a smooth external ray according to the rule $f\left(R^{t}\right)=$ $R^{d t}$.

For each exceptional argument $t \in \Sigma$, there exist a left hand limit ray $R^{t^{-}}$and a right hand limit ray $R^{t^{+}}$. Exceptional rays are also arcs running between the Julia set and infinity, though no longer smooth nor disjoint. They fail to be smooth at critical or pre-critical points. Pre-critical points are points that map, under some iterate of $f$, onto a critical point. Therefore, each exceptional ray contains at least one pre-critical or critical point. Moreover, a pre-critical or critical point in the basin of infinity belongs to at least 4 and at most finitely many exceptional 
rays. An exceptional ray $R^{t^{ \pm}}$maps either onto an exceptional ray $R^{d t^{ \pm}}$or onto the smooth ray $R^{d t}$. The notion of landing easily generalizes to right hand and left hand limit rays. It follows that if all the rays landing at $z$ are smooth, then all of the rays landing at $f(z)$ are smooth. The converse is not true.

When a point $z$ is exclusively the landing of smooth rays, we may define the type $A(z)$ of $z$ as the set of arguments of the external rays landing at $z$. The definitions of orbit portraits, sectors, angular size, etc., as well as their properties generalize to this context.

Here we are mainly interested in the points of $J(f)$ with infinite forward orbit. All of the rays, exceptional or not, that land at a point $z$ with infinite orbit must have irrational argument. Finiteness of the number of critical points and of the number of external rays which contain a given pre-critical or critical point implies that, for $k$ large enough, no exceptional ray lands at $f^{\circ k}(z)$. Thus we may apply the same argument of Section 4 to obtain the following result.

Theorem A.1. Consider a degree $d$ monic polynomial $f$ with disconnected Julia set $J(f)$. Let $z \in J(f)$ be a point with infinite forward orbit that is disjoint from the critical points of $f$. Let $k \geq 0$ be such that no right hand nor left hand limit ray lands at $f^{\circ k}(z)$. Then at most $d$ external rays land at $f^{\circ k}(z)$.

\section{ACKNOWLEDGMENTS}

This paper is a concise and revised version of Chapter 1 of the author's thesis Ki1. The author is grateful to John Milnor for his enlightening comments and suggestions during his graduate studies.

\section{REFERENCES}

[At] P. Atela, Bifurcations of dynamical rays in complex polynomials of degree two, Ergod. Th. \& Dynam. Sys. 12 (1992), 401-423. MR 94d:58128

[BL] A. Blokh and G. Levin, Growing trees, laminations and the dynamics on the Julia set, Preprint IHES, September 1999.

[CG] L. Carleson and T. W. Gamelin, Complex Dynamics, Springer-Verlag, 1993. MR 94h:30033

[D] A. Douady. Description of compact sets in C. In Topological Methods in Modern Mathematics. Publish or Perish, 1993. MR 94g:58185

[DH] A. Douady and J. H. Hubbard, Étude dynamique des polynomes complexes I, II, Publ. Math. Orsay, 1984-1985. MR 87f:58072a MR 87f:58072b

[G] L. R. Goldberg, Fixed Points of Polynomial Maps I, Ann. Scient. École Norm. Sup., $4^{e}$ série 25 (1992), 679-685. MR 94g:58107

[GM] L. R. Goldberg and J. Milnor, Fixed Points of Polynomial Maps II: Fixed Point Portraits, Ann. Scient. École Norm. Sup., $4^{e}$ série 26 (1993), 51-98. MR 95d:58107

[Ke] K. Keller. Invariant factors, Julia equivalences and the (abstract) Mandelbrot set. Lecture Notes in Mathematics, 1732. Springer-Verlag, Berlin, 2000. CMP 2000:13

[Ki1] J. Kiwi, Rational Rays and Critical Portraits of Complex Polynomials, Thesis, SUNY at Stony Brook, 1997. (Stony Brook IMS Preprint 1997/15)

[Ki2] J. Kiwi, Rational laminations of complex polynomials, pp 111-154 in Laminations and Foliations in Geometry, Topology and Dynamics, ed. M. Lyubich et al., Contemporary Mathematics 269, 2001. CMP 2001:08

[L] G. Levin, On backward stability of holomorphic dynamical systems, Fundamenta Mathematicae 158 (1998), 97-107. MR 99j:58171

[M1] J. Milnor, Dynamics in one complex variable: Introductory Lectures, Vieweg, 1999. CMP 2000:03 
[M2] J. Milnor, Periodic orbits, external rays and the Mandelbrot set: an expository account, pp 277-331 in Géométrie complexe et sytèmes dynamiques (Orsay, 1995), edited by M. Flexor et al., Astérique 261, 2000.

[Th] W. P. Thurston, On the combinatorics of iterated rational maps, Manuscript, 1985.

Facultad de Matemáticas, Pontificia Universidad Católica de Chile, Casilla 306, Correo 22, Santiago, Chile

E-mail address: jkiwi@mat.puc.cl 